\title{
Formation and evolution of dwarf elliptical galaxies: Structural and kinematical properties
}

\author{
S. De Rijcke ${ }^{1} \dagger$, D. Michielsen ${ }^{1}$, H. Dejonghe ${ }^{1}$, W.W. Zeilinger ${ }^{2}$ \\ and G.K.T. Hau ${ }^{3}$ \\ ${ }^{1}$ Sterrenkundig Observatorium, Ghent University, Krijgslaan 281, S9, B-9000 Gent, Belgium \\ email: sven.derijcke@UGent.be \\ ${ }^{2}$ Institut für Astronomie, Universität Wien, Türkenschanzstraße 17, A-1180 Wien, Austria \\ ${ }^{3}$ Department of Physics, South Road, Durham, DH1 3LE, UK
}

\begin{abstract}
We confront predictions of the currently popular theories for dE formation and evolution with the observed position of dEs in $\log L_{B}$ vs. $\log \sigma, \log L_{B}$ vs. $\log R_{\mathrm{e}}, \log L_{B}$ vs. $\log I_{\mathrm{e}}$, and $\log R_{\mathrm{e}}$ vs. $\log I_{\mathrm{e}}$ diagrams and in the $\left(\log \sigma, \log R_{\mathrm{e}}, \log I_{\mathrm{e}}\right)$ parameter space in which bright and intermediate-luminosity elliptical galaxies and bulges of spirals define a Fundamental Plane (FP). We show that the $\mathrm{dE}$ sequences in the various univariate diagrams are disjunct from those traced by bright and intermediate-luminosity elliptical galaxies and bulges of spirals. It appears that semi-analytical models (SAMs) that incorporate quiescent star formation with an essentially $z$-independent star-formation efficiency, combined with post-merger starbursts and the dynamical response after supernova-driven gas-loss, are able to reproduce the position of the $\mathrm{dEs}$ in the various univariate and bivariate diagrams.
\end{abstract}

Keywords. galaxies: dwarf - galaxies: fundamental parameters - galaxies: kinematics and dynamics

\section{Introduction}

For almost two decades now it has been known that dynamically hot galaxies (elliptical galaxies and bulges of spiral galaxies) are not scattered randomly in the three-dimensional space spanned by B-band luminosity ( $\log L_{B}$, expressed in solar B-band luminosities), half-light radius $\left(\log R_{\mathrm{e}}\right.$, expressed in kiloparsecs), and velocity dispersion $(\log \sigma$, expressed in $\mathrm{km} \mathrm{s}^{-1}$ ), but that instead they occupy a slender plane: the Fundamental Plane (FP) (Djorgovski \& Davis 1987, Dressler et al. 1987, Bender et al. 1992). Projections of the FP onto the coordinate planes, in combination with the particular way in which galaxies are distributed within the FP, produce the univariate relations between (i) luminosity and velocity dispersion $\left(\log L_{B}=\right.$ const. $+3.74 \log \sigma$, Faber \& Jackson 1976), (ii) luminosity and half-light radius $\left(\log L_{B}=\right.$ const. $+1.19 \log R_{\mathrm{e}}$, Fish 1963), (iii) surface brightness (expressed in solar B-band luminosities per square parsec) and half-light radius $\left(\log I_{\mathrm{e}}=\right.$ const. $-0.81 \log R_{\mathrm{e}}$, Kormendy 1977), (iv) and luminosity and surface brightness $\left(\log L_{B}=\right.$ const. $-1.46 \log I_{\mathrm{e}}$, Binggeli et al. 1984). The numerical values of these slopes and zero-points were taken from Guzmán et al. 1993, who made a compilation of these so-called fundamental relations for a sample of 66 Coma bright ellipticals.

The locus of the dEs and dS0s in the $\left(\log \sigma, \log R_{\mathrm{e}}, \log I_{\mathrm{e}}\right)$ space and in the univariate diagrams that require kinematical information, such as the $\sigma-L_{\mathrm{B}}$ relation, was up to now rather uncertain. After the early work by Nieto et al. 1990, Bender et al. 1992, and

$\dagger$ Postdoctoral Fellow of the Fund for Scientific Research - Flanders (Belgium)(F.W.O.) 
Guzmán et al. 1993, relatively little attention has been paid to the relation between the internal kinematics and the structural parameters of these faint, small elliptical galaxies and, particularly, to what we can learn from such relations regarding the origin and evolution of dEs. Still, explaining the scaling relations among the structural parameters of dwarf galaxies, which are believed to be the building blocks of more massive galaxies, is a crucial test for theories of cosmological structure formation. A comparison of theoretical predictions with observations can put strong constraints on the cosmological star-formation history and help to refine prescriptions for, e.g., star formation and energy feedback from supernova explosions, which play a crucial role in low-mass dwarf galaxies.

\section{Observations and data reduction}

Within the framework of an ESO Large Program, we observed deep major and minor axis spectra with unprecedented spatial and spectral resolution of a sample of $15 \mathrm{dEs}$ and dS0s, both in group (NGC5044, NGC5898, and NGC3258 groups) and cluster environments (Fornax cluster). We also collected Bessel VRI-band images of $22 \mathrm{dEs}$ and dS0s, including the $15 \mathrm{dEs} / \mathrm{dS} 0$ s of the spectroscopic sample. The data were obtained with the FORS2 imaging spectrograph mounted on the unit telescopes Kueyen and Yepun of the VLT.

From our images, we measured the surface-brightness profile, position angle, and ellipticity as a function of the geometric mean of major and minor axis distance denoted by $a$ and $b$ respectively, using our own software. The spectra, with typical exposure times of $5-8 \mathrm{~h}$ per position angle and a seeing in the range $0.3^{\prime \prime}-1.0^{\prime \prime} \mathrm{FWHM}$, cover the wavelength region around the strong CaII triplet absorption lines $(\sim 8600 \AA)$. We extracted the stellar kinematical information by fitting a weighted mix of late $\mathrm{G}$ to late $\mathrm{K}$ giant stars, broadened with a parameterised line-of-sight velocity distribution (LOSVD) to the galaxy spectra. We approximated the LOSVD by a fourth-order Gauss-Hermite series (Gerhard 1993, van der Marel \& Franx 1993). The strong CaII lines, which contain most of the kinematical information, are rather insensitive to the age and metallicity of an old stellar population (see e.g. Michielsen et al. 2003, Falcón-Barroso et al. 2003, Saglia et al. 2002), so template mismatch does not significantly affect our results. The spectra contain useful kinematical information out to $1.5-2 R_{\mathrm{e}}$.

\section{Univariate relations}

In the following, we confront the predictions of models for galaxy formation with the relations observed between the structural and kinematical parameters of dynamically hot galaxies. Semi-analytical models (SAMs) (Somerville et al. 2001, Nagashima \& Yoshii 2004) include the hierarchical merger tree that leads up to the formation of a galaxy of a given mass in a $\Lambda \mathrm{CDM}$ cosmology. SAMs make use of prescriptions for star-formation, energy feedback from supernova explosions, gas cooling, tidal stripping, dust extinction, and the dynamical response to starburst-induced gas ejection. Despite their simplicity, the models presented by Yoshii \& Arimoto 1987 already capture a lot of the physics of galaxy formation, at least in the mass-regime of the dEs, which apparently were assembled from what were still largely gaseous progenitors. In all figures, we will use them as a simple and instructive proxy for the physically more motivated SAMs. Alternatively, dEs could stem from late-type disk galaxies that entered the clusters and groups of galaxies about 5 Gyr ago $(z \sim 0.5)$. $N$-body simulations show that gravitational interactions trigger bar-formation in any small late-type disk galaxy (Scd-Irr) orbiting in a cluster (Moore et al. 1996, Moore et al 1998) or around a massive galaxy in a group environment 


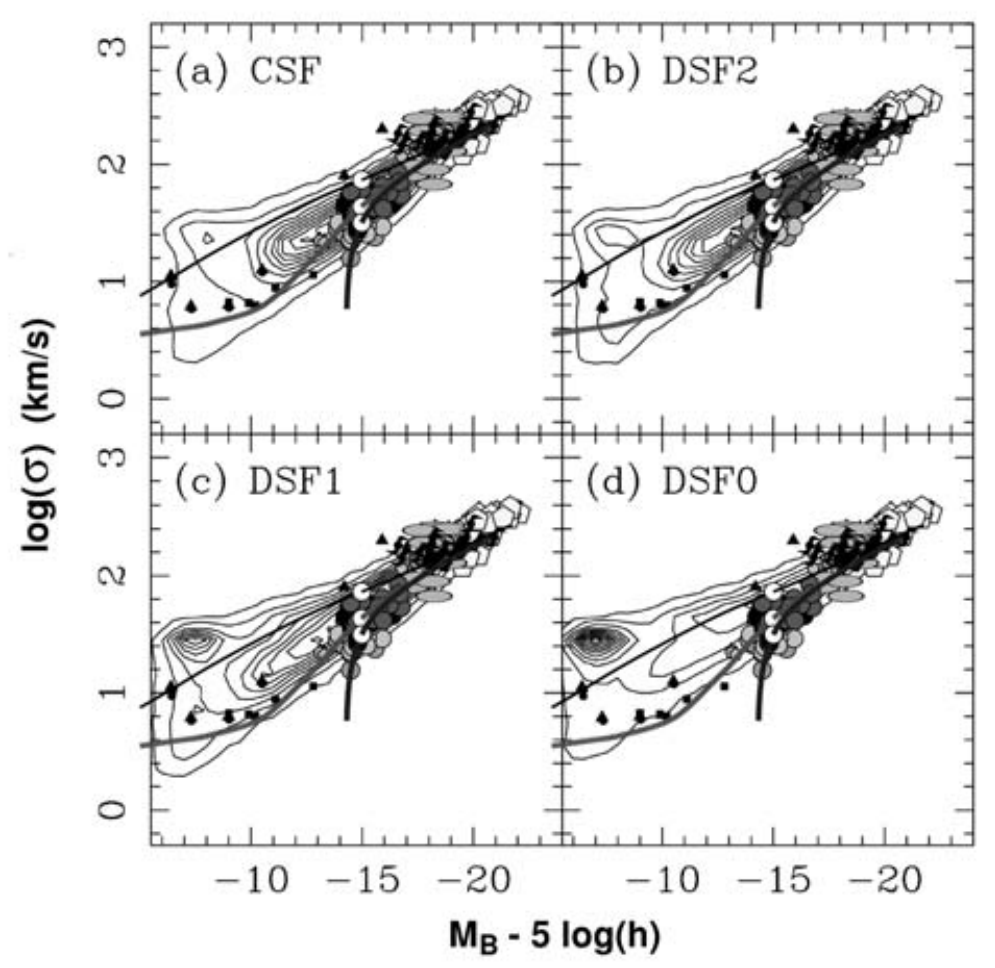

Figure 1. The luminosity $L_{B}$ vs. velocity dispersion $\sigma$ relation. The bright galaxies follow a trend $L_{B} \propto \sigma^{4}$ whereas the dwarf ellipticals follow a sequence defined by $L_{B} \propto \sigma^{1.6}$. This can be reproduced by the SAMs (contours) by allowing protogalaxies to expand after supernova-induced galactic winds following each major merger.

(Mayer et al. 2001) and strip large amounts of stars, gas, and dark matter from it by tidal forces. Internal dynamical processes, such as the buckling of the bar, subsequently transform a disk galaxy into a dynamically hot spheroidal dE within a timespan of a few Gyr (Mastropietro et al. 2004).

\subsection{The $\sigma-L_{\mathrm{B}}$ relation}

Bright and intermediate-luminosity ellipticals and bulges of spiral galaxies adhere closely to the $\sigma-L_{\mathrm{B}}$ relation: $L_{B} \propto \sigma^{\alpha}$, with $\alpha \sim 4$ (Faber \& Jackson 1976). As is obvious from Fig. 1, the $\sigma-L_{\mathrm{B}}$ relation becomes noticeably flatter below $M_{B} \sim-18.3$ mag. A straight-line fit to the dE data yields

$$
\log L_{B}=6.02^{ \pm 0.31}+1.57^{ \pm 0.19} \log \sigma .
$$

A $\mathrm{dE} \sigma-L_{\mathrm{B}}$ relation that is steeper than that of bright ellipticals is definitely excluded by these data. These results contradict the universality of the $\sigma-L_{\mathrm{B}}$ relation, which was suggested by Guzmán et al. 1993. A currently running project on the properties of faint early-type galaxies $\left(-22<M_{R}<-17.5\right)$ in the central 1deg of the Coma cluster yields a $\sigma-L_{\mathrm{B}}$ relation $L \propto \sigma^{2.01}$ at the faint end, which is consistent with our results (Matković \& Guzmán, private communication). 


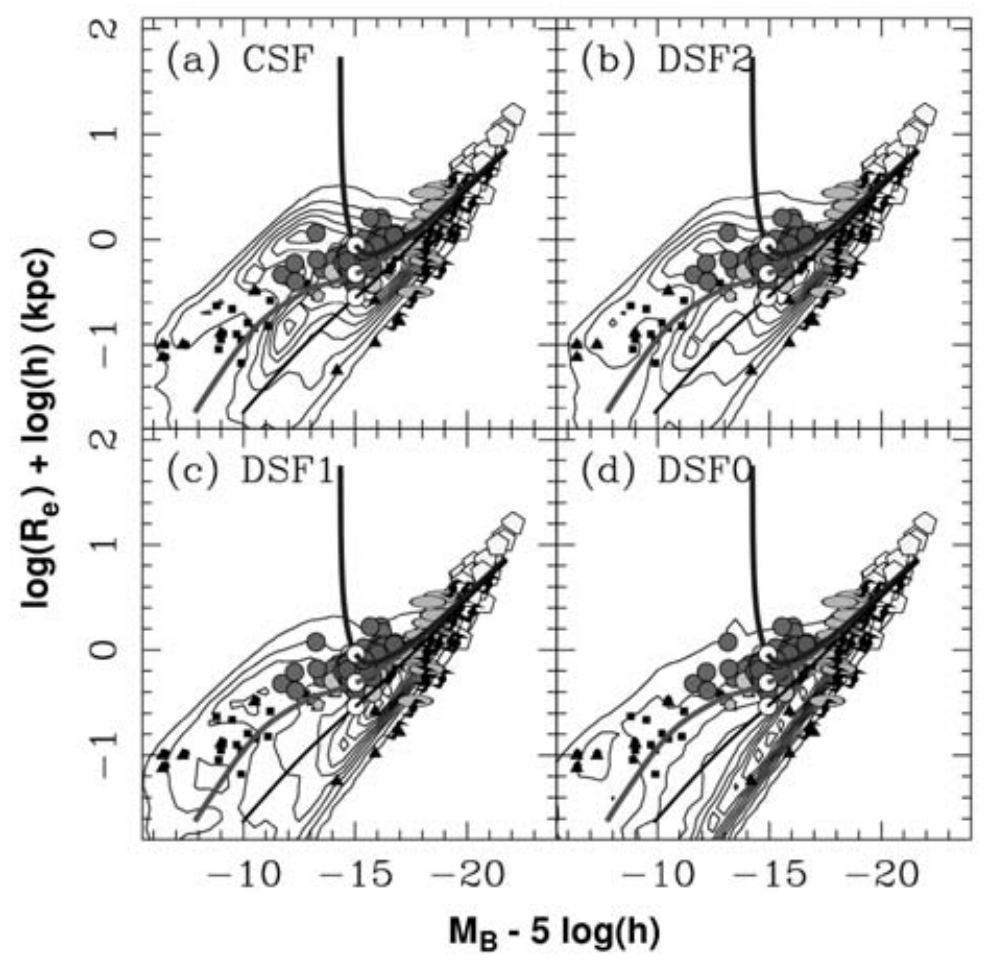

Figure 2. The luminosity $L_{B}$ vs. half-light radius $R_{\mathrm{e}}$ relation. Bright galaxies follow a well defined relation whereas dEs and dSphs are much more diffuse.

\subsection{The $R_{\mathrm{e}}-L_{\mathrm{B}}$ relation}

The simple fact that diffuse, low surface-brightness dEs exist puts strong constraints on the redshift dependence of the cosmic star-formation rate. Nagashima \& Yoshii 2004 have convincingly shown that structure-formation models in a $\Lambda$ CDM universe with a short star-formation timescale at high $z$ fail to produce such inflated dEs. In such a universe, $\mathrm{dEs}$ are assembled from progenitors that have already converted most of their gas into stars, and they are expected to trace the same sequences as the giant ellipticals, which are formed further down the merger tree from almost purely stellar progenitors, independent of the cosmic star-formation rate. Only models that have long enough star-formation timescales at high $z$, such that dEs can be formed by the mergers of gaseous progenitors, agree with these observations, e.g. the models of Nagashima \& Yoshii 2004 with a redshift independent star-formation time scale $\tau_{*}=1.3 \mathrm{Gyr}$, where the star-formation rate is given by $M_{\text {gas }} / \tau_{*}$ with $M_{\text {gas }}$ the Hi mass. The starburst triggered by each merger and the ensuing supernova-explosions eject gas, and thus lead to a population of diffuse dwarf galaxies with low velocity dispersions, as observed.

\section{Harassment}

These findings do not necessarily falsify the harassment scenario. The dEs observed so far overlap in $\kappa$-space with the present-day analogs of possible dE progenitors (the Scd and Irr galaxies). This overlap leaves open the possibility that we have observed both dEs that formed via hierarchical merging and dEs that formed via harassment. 
The dEs formed in these simulations are expected to rotate quite rapidly and some should still display some memory of their former state. Some of the dEs observed during the Large Program provide us with very strong evidence that harassment has indeed played an important role in their past evolution; this evidence includes embedded stellar disks (Barazza et al. 2002, De Rijcke et al. 2003a, Graham et al. 2003) or kinematically decoupled cores (De Rijcke et al. 2004).

\section{Conclusions}

Models for the evolution of dwarf and intermediate-luminosity elliptical galaxies, based on the idea that these stellar systems grow from collapsing primordial density fluctuations, are able to reproduce the observed relations between parameters that quantify their structure $\left(L_{B}, R_{\mathrm{e}}, I_{\mathrm{e}}\right)$ and internal dynamics $(\sigma)$ quite well. While dEs follow welldefined sequences in the various univariate diagrams, the correlations are not as tight as in the case of bright ellipticals. This cannot be due solely to measurement uncertainties, e.g. very deep photometry is now available that allows to determine $L_{B}$ and $R_{\mathrm{e}}$ with very small errors, still the scatter on the $\mathrm{dE} \log R_{\mathrm{e}}-\log I_{\mathrm{e}}$ relation is large. This cosmic scatter may be a consequence of the sensitivity of these low-mass systems to both internal (supernova explosions, feedback efficiency, the details of galactic winds, etc.) and external processes (gravitational interactions, tidal stripping of stars and ram-pressure stripping of gas, etc.) in group and cluster environments. Hence, these objects are ideal laboratories for studying these physical processes to which bright ellipticals seem to be quite insensitive.

\section{Acknowledgements}

Based on observations made at the European Southern Observatory, Chile (ESO Large Programme Nr. 165.N-0115). SDR wishes to thank Philippe Prugniel for fruitful discussions while visiting the CRAL-Observatoire de Lyon. WWZ acknowledges the support of the Austrian Science Fund (Project P14783). DM acknowledges the support of the Bijzonder OnderzoeksFonds (BOF Universiteit Gent)

\section{References}

Barazza, F.D., Binggeli, B. \& Jerjen, H. 2002, A\& $A$ 391, 823

Bender, R., Burstein, D. \& Faber, S.M. 1992, ApJ 399, 462

Binggeli, B., Sandage, A. \& Tarenghi, M. 1984, AJ 89, 64

De Rijcke, S., Dejonghe, H., Zeilinger, W.W. \& Hau, G.K.T. 2003, A\&A 400, 119

De Rijcke, S., Dejonghe, H., Zeilinger, W.W. \& Hau, G.K.T. 2004, A $\& A 426,53$

De Rijcke, S., Michielsen, D., Dejonghe, H., Zeilinger, W.W. \& Hau, G.K.T. 2005, A\&A, accepted Djorgovski, S. \& Davis, M. 1987, ApJ 313, 59

Dressler, A., Faber, S.M., Burstein, D., Davies, R.L., Lynden-Bell, D., Terlevich, R. \& Wegner, G. 1987, ApJ 313, L37

Faber S.M. \& Jackson R.E. 1976, ApJ 204, 668

Falcón-Barroso, J., Peletier, R.F., Vazdekis, A. \& Balcells, M. 2003, ApJ 588, L17

Fish R.A. 1963, AJ 68, 72

Geha, M., Guhathakurta, P. \& van der Marel, R.P. 2003, AJ 126, 1794

Gerhard, O.E. 1993, MNRAS 265, 213

Graham, A.W., Jerjen, H. \& Guzmán, R. 2003, AJ 126, 1787

Guzmán, R., Lucey, J.R. \& Bower, R.G., 1993, MNRAS 265, 731

Kormendy, J., 1977, ApJ 218, 333

Mastropietro, C., Moore, B., Mayer, L., Debattista, V.P., Piffaretti, R. \& Stadel, J. 2004, MN$R A S$ submitted, astro-ph/0411648 
Mayer, L., Governato, F., Colpi, M., Moore, B., Quinn, T., Wadsley, J., Stadel, J. \& Lake, G. 2001, ApJ 559, 754

Michielsen, D., De Rijcke, S., Dejonghe, H., Zeilinger, W.W. \& Hau, G.K.T. 2003, ApJ 597, L21

Moore B., Katz, N., Lake, G., Dressler, A. \& Oemler Jr., A. 1996, Nature 379, 613

Moore, B., Lake, G. \& Katz, N. 1998, ApJ 495, 139

Nagashima, M. \& Yoshii, Y. 2004, ApJ 610, 23

Nieto J.-L., Bender, R., Davoust, E. \& Prugniel, P. 1990, A\&\&A 230, L17

Saglia, R.P., Maraston, C., Thomas, D., Bender, R. \& Colless, M. 2002, ApJ 579, L13

Somerville, R.S., Primack, J.R. \& Faber, S.M. 2001, MNRAS 320, 504

van der Marel, R.P. \& Franx, M. 1993, ApJ 407, 525

van Zee, L., Skillman, E.D. \& Haynes, M.P. 2004, AJ 128, 121

van Zee, L., Barton, E.J. \& Skillman, E.D. 2004, AJ 128, 2797

Yoshii, Y. \& Arimoto, N. 1987, A\&A 188, 13

\section{Discussion}

PRUGNIEL: Why do you say that disks are evidence for harassment?

De RiJCKE: As was pointed out by Moore et al. (1998) and Mastropietro at al. (2004), harassment not always completely transforms a late-type galaxy into a dwarf elliptical. In fact, most of the simulated dEs end up having embedded disks, bars, spiral arms, etc. So our observations corroborate this idea.

FOnT: Regarding the $\sigma-L$ relation: it seems that the theoretical models you presented cannot fit the low- $\sigma$ (hence low-mass) regime well. One would expect that for low-mass galaxies winds play an important role, yet neither of the mass ejection models seem to fit the data. Are these theoretical models reliable? Do they take into account environmental effects?

DE RiJCKE: These models rely on an empirical relation between total mass and binding energy. Since gas is ejected when it is heated above the escape velocity, this is clearly an important ingredient of the models. The empirical relation between mass and binding energy does, however, not provide a good description of dwarf spheroidals, so this may be the reason the models fail to reproduce the properties of the dSphs. More recent semianalytic models for galaxy formation through hierarchical merging give a much better description of the $\mathrm{dSph}$ properties. None of these models take into account environmental effects. 